\title{
WHITE DWARFS AND PLANETARY CENTRAL STARS
}

\author{
James Liebert \\ Steward Observatory \\ University of Arizona \\ Tucson, Arizona 85721, USA
}

\begin{abstract}
Studies of hot white dwarf samples constrain the properties and evolution of planetary nuclei and the nebulae. In particular, the white dwarf and planetary nebulae formation rates are compared. I discuss the overlap of the sequences of white dwarfs having hydrogen (DA) and helium-rich (DO) atmospheres with known central stars of high surface gravity. There is evidence that the hydrogen atmosphere nuclei have "thick" outer hydrogen layers $\left({ }^{2} 10^{-4} M_{\odot}\right)$, but that DA white dwarfs may have surface hydrogen layers orders of magnitude thinner. Finally, a DA planetary nucleus is discussed $(0950+139)$ which has undergone a late nebular ejection; this object may be demonstrating that a hydrogen layer can be lost even after the star has entered the white dwarf cooling sequence.
\end{abstract}

\section{STUDIES OF HOT WHITE DWARFS: CONSTRAINTS ON THE PLANETARY NEBULA EVOLUTION}

It is now commonly accepted that the principal "channel" into the white dwarf sequence is post-asymptotic giant branch (post-AGB) evolution with the ejection of a planetary nebula. It follows that the studies of samples of hot white dwarfs are relevant to the masses and compositions of planetary nuclei, their evolution, and the formation rate and evolution of the nebulae. Harry Shipman has summarized nicely some of the ongoing scientific questions raised in studies of the degenerate dwarfs. We shall revisit the question of whether and how the surface abundances in white dwarfs change with cooling age in addressing how the central stars (CSPN) evolve into white dwarfs.

\subsection{Comparison of the Birthrates}

The formation rate of white dwarf stars should be an upper limit to the planetary nebula formation rate, but close to the actual value, provided one can sample objects from the same galactic population. Yet a recent comprehensive determination of the white dwarf birthrate (Fleming, Liebert and Green 1986) yields a number which is substantially smaller than most published estimates for planetary nebulae. It is approximately 545

S. Torres-Peimbert (ed.), Planetary Nebulae, 545-554.

(c) 1989 by the IAU. 
a factor of two lower than the value for the nebular formation rate favored by Phillips (these proceedings).

Hot white dwarfs may be found in complete, color and magnitude limited surveys, such as the Palomar Green Survey (Green, Schmidt and Liebert 1986). Because these stars may be readily assigned photometric or spectroscopic parallaxes, and because the cooling evolution for the hotter stars is based on relatively simple and well understood physics, relatively accurate space densities and birthrates for these stars should be derivable. It is arguably more difficult to construct a complete sample, and assign distances and nebular lifetimes to the planetary nebulae.

On the other hand, the white dwarf number derived from an ultraviolet excess survey does not include those degenerates in binary systems with companions more luminous than $M$ dwarfs. We have already heard strong evidence from Bond and Mendez that the CSPN include significant fractions of binary systems. Moreover, the white dwarf number is drawn from a sample at typical distances of $\sim 100 \mathrm{pc}$ with an indicated scale height (from evidence other than the Palomar Green Survey) in the 250-350 pc range. The planetary samples generally cover distances of order $1 \mathrm{kpc}$, and Phillips has reviewed the evidence that their scale height is closer to 150 pc. A local population might be more affected by inhomogeneities in the space density of the disk population. For these reasons, it is not clear that the preferred planetary number is wrong, even though the white dwarf result should be a fairly accurate determination for the sample that it represents.

\subsection{Targets of Opportunity in Searches for Residual Nebulae}

Samples of hot white dwarfs and field subdwarfs have recently been the targets of sensitive searches for residual nebulae. Such surveys for faint, extended emission lines are potentially useful in comparing the evolutionary time of the CSPN with that for dispersal of the nebula. This may permit a testing of the validity of "kinematic ages" and the identification of a few rapidly-evolving CSPN of high core mass. However, it is also becoming clear (see Section 3) that nebulae can exist around white dwarfs which result from an episode of mass loss by the star long after it has left the AGB.

In the last few years, several CSPNs have been found to have white dwarf surface gravities based on spectra and model atmospheres analyses. Certainly the majority of these were studied because of the prior discovery of a nebula. A few previously-known field white dwarfs have shown evidence for residual nebulosity, though the largest imaging survey of known hot subdwarfs and white dwarfs (to my knowledge) by Kwitter and Massey (1987) and other collaborators has yet to yield a definite detection of a nebula. A smaller set observed by Reynolds (1987) has yielded one interesting candidate. Some of the successful matchups of nebula and white dwarf CSPN are highlighted in the next section. 
2. MATCHING UP THE SEQUENCES OF H AND HE-RICH CSPN AND WHITE DWARFS

Planetary nebulae are relatively rare objects, due to the relatively short nebular lifetimes, and can be found in significant numbers only at distances relatively large in comparison to those for samples of white dwarfs. The degenerate stars are of course sufficiently faint that they cannot be found easily at distances beyond a few hundred parsecs. Accordingly, some low luminosity CSPN in known nebulae may be too faint to be detected or studied. The detection of very faint, residual nebulae around nearby, hot degenerate stars offers a way of bridging this luminosity gap and seeing how well the CSPN and white dwarf sequences match up, despite the differing discovery techniques and search volumes. In particular, since the relative frequencies of hydrogen and helium-dominated atmospheric compositions are approximately $80 \%$ to $20 \%$ (respectively) for both the CSPN and the hot white dwarfs, it is logical to start with the assumption that the dominant atmospheric constituent does not change as the CSPN evolve into white dwarfs. This can be tested by looking for continuity and overlap at the CSPN/white dwarf H-R Diagram boundary for both composition sequences.

\subsection{The He-Rich CSPN/White Dwarf Sequence}

For the minority He-rich post-AGB stars, there is ample evidence for continuous evolution across this boundary. At or just above the hot end of the helium atmosphere white dwarf sequence lies the group of pulsating stars whose prototype is PG1159-035 (GW Vir). These have effective temperatures near to or exceeding $100,000 \mathrm{~K}$, surface gravities near white dwarf values (log $g \sim 7$ ) and photospheric lines of He $11, C$ IV and $\mathrm{O}$ VI. A nonpulsating object $\mathrm{H} 1504+65$ discussed by Harry Shipman appears to be even hotter (Nousek et al. 1986). Several more field stars with similar spectra apparently do not pulsate (Grauer et al. 1987). Searches for residual nebulosity by $K$ witter and collaborators have apparently yielded only null results so far.

That these objects nevertheless invade the domain of He-rich CSPN is indicated by the similar pulsating nucleus of $\mathrm{K} 1-16$ (Grauer and Bond 1984) and possibly the nucleus of VV47 (Liebert et al. 1988a). They have strong spectroscopic similarities to the CSPN of the "O VI" type (Sion, Liebert and Starrfield 1985). Some of these show vigorous, photospheric mass loss in the form of Wolf-Rayet emission features, although this activity has abated in some of the hottest helium-rich CSPN (Mendez et al. 1986). In fact the K1-16 nucleus shows weak $0 \mathrm{VI}$ $3811,3830 \AA$ emission; the ultraviolet spectrum also indicates that mass loss is ongoing (Kaler and Feibelman 1985).

The planetary nebula link could extend downward in the H-R Diagram into the region of the hot DO degenerates, which show primarily lines of He II. Reynolds (1987) has found evidence for very extended nebulosity around PG0108+101. Wesemael, Green and Liebert (1985) estimate that this star has $\mathrm{T}$ eff $\sim 80,000 \mathrm{~K}, \log \mathrm{g}>7$, and $\mathrm{He} / \mathrm{H} \geq=2$ by number. In 
Kaler's talk, the spectrum presented for the nucleus of In 1 appears also to be that of a hot, helium-rich star of high surface gravity.

In summary, a broad overlap is developing between the sequences of known He-rich CSPN and the samples of He-rich degenerate stars. It is logical to hypothesize that a continuous evolutionary sequence of decreasing luminosity and stellar mass loss carries the $\mathrm{WC} / \mathrm{O} \mathrm{VI}$ nuclei into the pulsational instability strip and that they then cool into DO white dwarfs.

\subsection{The Hydrogen Rich Sequence}

A few known $\mathrm{H}$-rich CSPN have temperatures and gravities which also overlap the hot end of the sequence of DA white dwarfs. The best analyzed cases are the nuclei of Abell 7 and NCC 7293 (Mendez, Kudritzki and Simon 1983). The former has a Teff "75,000 K, $\log g \approx 7$ while estimates for the latter are approximately 90-100,000 K and log $\mathrm{g} \sim$ 6.5-7 (see also Mendez' talk, this meeting). PG0950+139, discussed in the next section, also has a temperature near $70,000 \mathrm{~K}$ and a surface gravity (as indicated by broader lines) higher than than for the Abell 7 nucleus.

Yet the field DA stars do not extend continuously up to Teff $100,000 \mathrm{~K}$. The hottest known field white dwarfs with H-rich compositions and $\log \mathrm{g} z=7$ appear to have temperatures near $70,000 \mathrm{~K}$ (Holberg 1987). Below 70,000 K, the DA stars constitute a large fraction $(\sim 80 \%)$ of all hot white dwarfs. Thus it is reasonable to ask why the helium sequence includes hot DO stars near 80,000 K and even hotter objects of high gravity past $100,000 \mathrm{~K}$, while there are very few if any such hot counterparts in the majority $\mathrm{H}$-rich sequence.

\subsection{The $H$ Layer Masses and Evolutionary Lifetimes}

The lack of DA stars with $T_{\text {eff }}>80,000 \mathrm{~K}$ may be due to the fact that the lifetimes in the $\mathrm{H}$-rich CSPN phase are much shorter, provided that these objects have thick enough outer hydrogen layers that they leave the $A G B$ as $H$ shell-burning objects. Schönberner $(1981,1986$, and this conference) has advanced two arguments that most CSPN are hydrogen burning objects:

(1) The gap in the absolute magnitude distribution of CSPN near $M v=+5$ divides PNNs into high and low luminosity groups, and is attributed to the onset of rapid evolution as the $H$ shell source shuts off at the end of the luminous phase of horizontal evolution in the H-R diagram. In particular, Schönberner's tracks for helium shell-burning CSPN are not compatible with the existence of such a gap;

(2) A similar argument can be made in a distance-independent way, using the nebular excitation parameter, and Kaler's sample of large (old) CSPN (see also Schmidt-Voigt and Köppen 1987).

It follows that such PNNs with thick outer hydrogen envelopes 
should enter the white dwarf stage as DA stars. The models of Koester and Schönberner (1986) and of Iben and Tutukov (1984) predict that the phase of rapid evolution for those objects with quenched $H$ shells should last until the CSPN reach approximately $\log L / L_{O} \sim 2$ (see Iben and Tutukov, Fig. 1). This corresponds to the approximate luminosities of the PG1159-035/H1504+65 objects and may account for the scarcity of $\mathrm{H}$-rich counterparts. In fact the observed beginning of the $D A$ sequence at $\log L / L_{O} \sim 1(70,000 \mathrm{~K})$ suggests that the phase of rapid evolution lasts to lower luminosities than predicted, although the observed samples are small. Moreover, there should be some allowance for the uncertainties in the derived stellar L, Teff parameters and in the interiors models.

\subsection{Do DA White Dwarfs Have Very Thin Outer Hydrogen Layers?}

In any case, the distributions of the hottest $H$ and He-rich objects forming white dwarfs are explainable only if the former retain thick hydrogen shells $\left(M_{H} Z=10^{-4} M_{\odot}\right)$, a necessary condition for the luminous $H$ shell-burning phase. The distributions of these low luminosity stars are thus consistent with and support Schönberner's arguments concerning the majority of CSPN.

There are several arguments, however, which lead to the conclusion that DA white dwarfs have outer layer masses many orders of magnitude thinner than $10^{-4} \mathrm{M}_{\odot}$. These arguments are summarized in Fontaine and Wesemael (1987), and are discussed in other review papers presented at IAU Colloquium 95. Four of the major arguments are outlined below:

1. He/H abundance ratios observed in hot DA stars $(>30,000 \mathrm{~K})$ are orders of magnitude too large to be explained by radiative levitation of helium (Vennes et al. 1987). These authors favor as the origin a diffusion tail reaching upwards to the surface through very thin hydrogen layers of only $10^{-13}$ to $10^{-16} \mathrm{M}_{\odot}$.

2. There are currently no known white dwarfs with He-rich atmospheres in the temperature range 45,000< Teff $<30,000 \mathrm{~K}$ (Liebert et al. 1986). Yet further statistics indicate that some $25 \%$ of the $D A$ turn into He-rich atmospheres below $30,000 \mathrm{~K}$ (Sion 1984). A possible explanation of this peculiar observational result, due primarily to $G$. Fontaine, is that convective overshooting in the helium envelope could mix away an H-rich surface layer, provided that the latter consisted of less than approximately $10^{-15} \mathrm{M}_{\odot}$. Shipman, in the previous talk, suggests that the "DB gap" might be explainable instead by differences in the cooling times of the two sequences.

3. The pulsating $Z Z$ Ceti stars include all or nearly all $D A$ degenerates cooling through the $10-13,000 \mathrm{~K}$ instability strip. The mode-trapping model for the pulsational driving favored to produce the rather long periodicities observed in these objects requires modest hydrogen layer masses of order $10^{-8} \mathrm{M}_{\odot}$ or less (Winget et al. 1982). However, this conclusion is disputed by Cox et al. (1987). 
4. Finally, it is known that most of the remaining DA stars must evolve into objects with helium atmospheres $T_{\text {eff }}<10,000 \mathrm{~K}$. In fact Winget et al. (1982) and others have argued that the mixing due to penetration of the helium layer by the hydrogen convective zone may terminate the $Z Z$ Ceti instability strip. In Greenstein's (1986) compilation of high signal-to-noise ratio observations, it is apparent that some $70 \%$ of the stars have become helium-rich at the temperatures approaching the low extreme $(\sim 6,000 \mathrm{~K})$ that hydrogen lines would be visible. The calculations indicate again (D'Antona and Mazzitelli 1979) that convective mixing will not take place unless the outer hydrogen layer is thinner than approximately $10^{-6} \mathrm{M}_{\odot}$.

Individually, these arguments for thin outer hydrogen layers depend on the assumptions that we understand accurately enough the physics of (1) envelope convection, especially the behavior in regions having large gradients in the chemical composition, (2) selective radiative acceleration processes and their competition against gravitational and thermal diffusion, and/or (3) pulsational driving theory. Individually, each argument may not be very convincing. Together, they constitute a case which must be taken seriously. On balance, the observational evidence that the fraction of DA white dwarfs decreases markedly with decreasing surface temperature is difficult to explain if the majority of these stars retain outer layer masses of hydrogen of order $10^{-4} \mathrm{M}_{\mathrm{O}}$.

Fontaine and Wesemael (1987) suggest that all DA stars have very thin outer envelopes, and that the most logical explanation of the absence of DA stars near $100,000 \mathrm{~K}$ is that the pulsating helium-rich and the hot DO stars retain enough residual envelope hydrogen for this later to float to the surface, form a thin outer hydrogen layer, and convert the He-rich atmosphere into a DA star.

This simple hypothesis has difficulty in accommodating the arguments that the majority $\mathrm{H}$-rich $\mathrm{PN}$ nuclei must have thick hydrogen layers in order to undergo hydrogen shell burning. At the low luminosity end, one wonders how the H-rich CSPN would lose these thick layer masses and turn into $\sim 10^{5} \mathrm{~K}$ pulsating He-rich objects just after the stars have evolved rapidly through the $M_{V} \sim+5$ region; the corresponding mass loss rates would exceed $10^{-8} \mathrm{M}_{\odot}$ per year for typical masses near $0.6 \mathrm{MO}$. In particular, low luminosity hydrogen-rich CSPN such as Abell 7 are most difficult to understand. However, the object discussed in the next section offers a clue that this process of converting a thick CSPN envelope into a thin DA white dwarf envelope may actually happen!

\section{EGB/PG 0950+139: EVIDENCE FOR ONGOING/RECENT MASS LOSS FROM A HOT DA WHITE DWARF}

One of the very hottest known DA white dwarfs is associated with an extended planetary nebula of low surface brightness. Ellis, Grayson and Bond (1984), EGB) identified nebulosity of roughly circular morphology 
some 12 arc minutes in diameter around a 15th magnitude blue star. The stellar counterpart was independently catalogued in the Palomar Green Survey as PG 0950+139. Both sets of authors were aware of a compact nebular line spectrum, essentially unresolved in a two-dimensional Palomar 5-m SIT Vidicon scan (by R. Green), and in two additional spectra with the Kitt Peak National Observatory 4-m telescope and cryogenic camera/CCD system (by H. Spinrad and R. Green). The extended nebula is so faint that it is not detected in $H$ or [O III] lines on any of these long slit spectra. H. Bond (private communication) comments that this component has highest surface brightness near an outer rim. The analysis of both the compact nebular component and the stellar photospheric spectrum is described in a forthcoming paper (Liebert et al. 1988b).

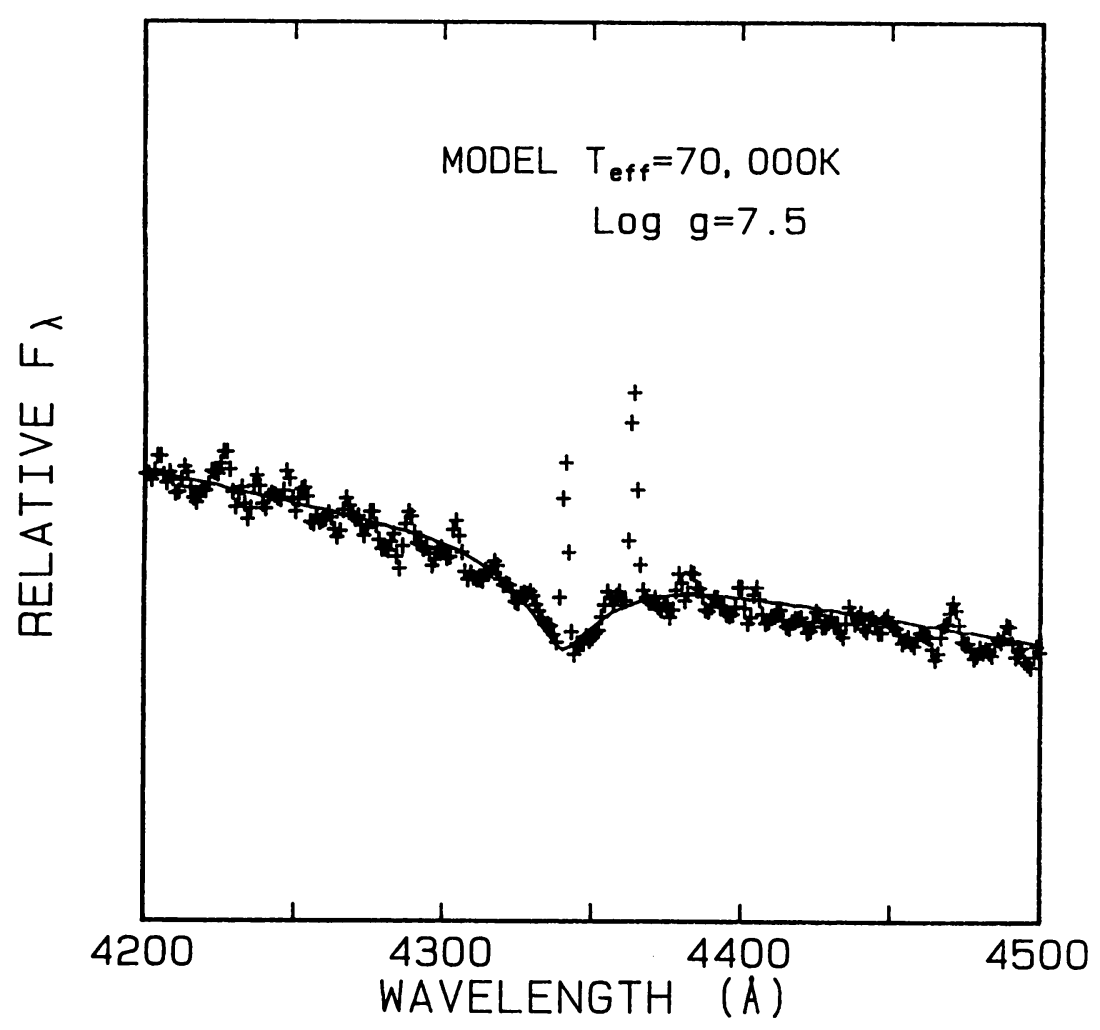

Figure 1. The spectrum of the photosphere of 0950+139 centered on the $\mathrm{H} \gamma$ line with the synthetic spectrum of a pure hydrogen, $\mathrm{T}_{\text {eff }}=70,000$, log $g=7.5$ model by $F$. Wesemael (courtesy of collaborators J. Holberg and K. Kidder). Nebular emission lines of $H_{\gamma}\left[\begin{array}{lll}O & 111\end{array}\right] 4363 \AA$ and $H e ~ I$ $4471 \AA$ are also visible. 


\subsection{Properties of the DA Central Star}

The nucleus of this planetary is a white dwarf at the high temperature extreme of the DA distribution. J. Holberg and K. Kidder have analyzed the photospheric $\mathrm{H}$-gamma and other absorption lines. The best fit using pure hydrogen, LTE models by $F$. Wesemael is T eff $\sim 70,000( \pm 7,000 \mathrm{~K})$, $\log \mathrm{g} \sim 7.5( \pm 0.25)$, and $\mathrm{H} / \mathrm{He}>1$. The helium abundance is not estimated because of the problem of nebular contamination of any narrow He II 4686A absorption. The star has broader hydrogen lines (i.e., higher log $\mathrm{g}$ by +0.5 ) than the CSPN of Abell 7, which has a similar Teff

\subsection{Properties of the Unresolved Nebular Component}

The compact nebula is quite remarkable. Detectable forbidden lines of [O III], [Ne III] and [O I] are seen, along with permitted lines of $H$ and possibly He. The flux ratio usually used in calculating the [O III] temperature provided the first clue to the unusual physical conditions. The ratio

$$
R[0 \quad I I I]=[f(5007 \AA)+f(4959 \AA)] / f(4363 \AA)
$$

was found to be only 10.9, while typical planetary nebulae values are as much as an order of magnitude larger. Even for ion densities approaching $\mathrm{N}_{e}=10^{6} \mathrm{~cm}^{-3}$, this value leads to very high electron temperature $\left(T_{e}\right)$ estimates, in excess of $20,000 \mathrm{~K}$.

Normally the density of a nebular region may be estimated from the ratios of doublets of $[0 \quad 11]$ or $[S$ II], but these lines do not appear. Yet the very large $\left[\begin{array}{ll}0 & I I I\end{array}\right] /\left[\begin{array}{ll}0 & 11\end{array}\right]$ excitation ratio is inconsistent with a similar measure of nebular excitation using permitted lines, the ratio of the $\mathrm{He}$ II $4686 \AA$ to the $H-\beta \quad 4861 \AA$ lines. Even stranger at first glance is the appearance of $\left[\begin{array}{lll}0 & 1\end{array}\right] 6300,6360$ auroral lines in two red CCD spectra.

lonized gas at densities above $10^{6} \mathrm{~cm}^{-3}$ may occur in the line-emitting regions of active galactic nuclei and radio galaxies. In this context, the critical densities at which quenching of various forbidden lines occurs are listed in Fillipenko (1985, Table 3). For example, $\mathrm{Ne}$,crit for the [O III] 4959 and 5007 transitions occurs at 7.9 $\times 10^{5} \mathrm{~cm}^{-3}$, while the value for [O III] 4363 is much higher at $3 \times 10^{7}$.

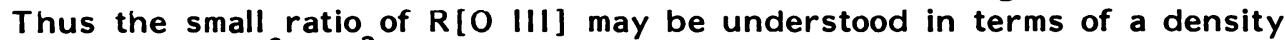
higher than $10^{6} \mathrm{~cm}^{-3}$. The unseen [O II], [N II] and [S II] doublets are all quenched at densities below $10^{5} \mathrm{~cm}^{-3}$. The visible [ $\mathrm{Ne}$ III] and [ 0 1] transitions survive at higher densities, the former at $1.2 \times 10^{7}$ $\mathrm{cm}^{-3}$, while the weak [ 0 I] lines suggest that some material in the inner nebula may have densities as low as $1.2 \times 10^{6} \mathrm{~cm}^{-3}$.

On balance, we argue that the mean density of the unresolved nebular component of $0950+139$ is near $10^{6} \mathrm{~cm}^{-3}$. Using this number, $R\left[O \quad\right.$ III] yields an estimate of $T_{e} \sim 19,100 \mathrm{~K}$, still quite high. Assuming a fully-ionized, uniform spherical nebula at this single 
density and temperature, we estimate a nebular mass of order $10^{-8} \mathrm{M}_{0}$, and a radius of order $10 \mathrm{a} . \mathrm{u}_{\text {.! }}$ There is no indication that the helium abundance is high, though the abundance constraints on He and heavier elements are sparse.

These results lead to the inescapable conclusion that the mass loss has been a recent or ongoing event. While the expansion rate of this component is as yet unknown, velocities of $10-50 \mathrm{~km} / \mathrm{s}$ would extend the material to $10 \mathrm{a} . \mathrm{u}$. in 1-10 years. The cooling age of a $0.6 \mathrm{M}_{\odot}$ white dwarf at $70,000 \mathrm{~K}$ is approximately $5 \times 10^{5}$ years!

\subsection{0+139: A Case of Envelope "Thinning"?}

These results suggest the possibility that significant mass loss may evaporate most or all of the outer hydrogen envelope as the stars approach and enter the white dwarf stage. Since this CSPN is one of the few known DA white dwarfs near the $70,000 \mathrm{~K}$ limit of the known sequence, the frequency of observable nebulae ejections around such objects is not determined. However, one may estimate that an average mass loss rate of $10^{-10} M_{\odot}$ per year would be sufficient to reduce the outer layer mass to nil, if the instability lasted for the approximately $10^{6}$ years required for a white dwarf to cool to $60,000 \mathrm{~K}$. Steady mass loss rates this small are difficult to exclude, even for stars observed with the International Ultraviolet Explorer Satellite at high resolution. It is also possible that such mass loss may occur in the form of sporadic ejections, so that most objects may not show readily detectable nebular emission at any given time. However, there are no detected changes between nebular spectra obtained in the late 1970 s and those obtained in the mid-1980's.

This discovery should spur further attempts to detect residual nebulosity around hot DA white dwarfs, and evidence for ongoing mass loss around hydrogen-rich CSPNs with known extended components. Further identifications of the rare DA white dwarfs near the high temperature limit may be necessary in order to determine the frequency of the late mass loss phenomenon observed in 0950+139. Moreover, a helium-rich DO star of similar temperature also shows some evidence for recent mass loss (Downes et al. 1987).

I acknowledge support from the National Science Foundation (grant AST 85-14778). I thank J. Holberg and K. Kidder for providing Fig. 1.

\section{REFERENCES}

Cox, A. N., Starrfield, S. G., Kidman, R. B. and Pesnell,D.: 1987, Astrophys. J., 317, 303.

D'Antona, F. and Mazzitelli, I.: 1979, Astron. Astrophys., 74, 161. Downes, R. A., Sion, E. M., Liebert, J. and Holberg, J.: 1987, Astrophys. J., , 321, 943. 
Ellis, G. L., Grayson, E. T. and Bond, H. E.: 1984, Publ. Astron. Soc. Pacific, 96, 283.

Fillipenko, A. V.: 1985, Astrophys. J., 289, 475.

Fleming, T. A., Liebert, J. and Green, R. F.: 1986, Astrophys. J., 308, 176.

Fontaine, G. and Wesemael, F.: 1987, A. G. D. Philip, D. S. Hayes and J. Liebert (eds.), 'Second Conference on Faint Blue Stars,' IAU Coll. 95 (in press).

Grauer, A. D. and Bond, H. E.: 1984, Astrophys. J., 277, 211.

Grauer, A. D., Bond, H. E., Green, R. F., Liebert, J. and Fleming, T. A.: 1987, Astrophys. J., (in press).

Green, R. F., Schmidt, M. and Liebert, J.: 1986, Astrophys. J. Suppl., $61,305$.

Greenstein, J. L.: 1986, Astrophys. J., 304, 334.

Holberg, J.: 1987, A. G. D. Philip, D. S. Hayes and J. Liebert (eds.), 'Second Conference on Faint Blue Stars,' IAU Coll. 95, (in press) and private communication.

Iben, I., Jr. and Tutukov, A. V. 1984, Astrophys. J., 282, 615.

Kaler, J. B. and Feibelman, W. A.: 1985, Astrophys. j., 297, 724.

Koester, D. and Schönberner, D.: 1986, Astron. Astrophys., 154, 134.

Kwitter, K. and Massey, P. 1987, in preparation.

Liebert, J.,Fleming, T. A., Green, R. F. and Grauer, A. D.: 1988a, submitted to Publ. Astron. Soc. Pacific.

Liebert, J., Green, R. F., Bond, H. E., Holberg, J., Kidder, K., Fleming, T. A. and Wesemael, F.: 1988b, preprint.

Liebert, J., Wesemael, F., Hansen, C. J., Fontaine, G., Shipman, H. L., Sion, E. M., Winget, D. E. and Green, R. F.: 1986, Astrophys. J., $309,241$.

Mendez, R. H., Kudritzki, R. P. and Simon, K. P.: 1983, D. R. Flower (ed.), 'Planetary Nebulae,' IAU Symp. 103, 343.

Mendez, R. H., Miguel, C. H., Heber, U. and Kudritzki, R.: 1986, K. Hunger, D. Schönberner and N.K. Rao (eds.), 'Hydrogen Deficient Stars and Related Objects,' IAU Coll. 87, 323.

Nousek, J. A., Shipman, H. L., Holberg, J. B., Liebert, J., Pravdo, S. H., White, N. E. and Giommi,P.: 1986, Astrophys. J., 309, 230.

Reynolds, R. J.: 1987, Astrophys. J., 315, 234.

Schmidt-Voigt, H. and Köppen, J.: 1987, Astron. Astrophys., 174, 223.

Schönberner, D.: 1981, Astron. Astrophys.., 103, 119.

Schönberner, D.: 1986, Astron. Astrophys., 169, 189.

Sion, E. M.: 1984, Astrophys. J., 282, 612.

Sion, E. M., Liebert, J. and Starrfield, S. G.: 1985, Astrophys. J.. 292, 471.

Vennes, S., Pelletier, C., Fontaine, G. and Wesemael, F.: 1987, A. G. D. Philip, D. S. Hayes and J. Liebert (eds.), 'Second Conference on Faint Blue Stars,' IAU Coll. 95 (in press).

Wesemael, F., Green, R. F. and Liebert, J.: 1985, Astrophys. J. Suppl., 58,379 .

Winget, D. E., Van Horn, H. M., Tassoul, M., Hansen, C. J., Fontaine, G. and Carroll, B. W.: 1982, Astrophs. J. Letters, 252, L65. 